\title{
On the efficiency of PAPR reduction schemes deployed for DRM systems
}

\author{
Sanam Moghaddamnia ${ }^{1 *}$, Albert Waal ${ }^{2}$, Martin Fuhrwerk ${ }^{1}$, Chung Le ${ }^{1}$ and Jürgen Peissig ${ }^{1}$
}

\begin{abstract}
Digital Radio Mondiale (DRM) is the universally, openly standardized digital broadcasting system for all frequencies including LW, MW, and SW as well as VHF bands. Alongside providing high audio quality to listeners, DRM satisfies technological requirements posed by broadcasters, manufacturers and regulatory authorities and thus bears a great potential for the future of global radio. One of the key issues here concerns green broadcasting. Facing the need for high-power transmitters to cover wide areas, there is room for improvement concerning the power efficiency of DRM-transmitters. A major drawback of DRM is its high peak-to-average power ratio (PAPR) due to the applied transmission technology based on Orthogonal Frequency Division Multiplexing (OFDM), which results in non-linearities in the emitted signal, low power efficiency, and high costs of transmitters. To overcome this, numerous schemes have been investigated for reducing PAPR in OFDM systems. In this paper, we review and analyze various technologies to reduce PAPR providing that the technical feasibility and DRM-specific system architecture and edge conditions regarding the system performance in terms of modulation error rate, compliance with frequency mask, and synchronization efficiency are ensured. All evaluations are carried out with $1 / Q$ signals which are monitored in real operation to present the actual performance of proposed PAPR techniques. Subsequently, the capability of the best approach is evaluated via measurements on a DRM test platform, where achieved transmit power gain of $10 \mathrm{~dB}$ is shown. According to our evaluation results, PAPR reduction schemes based on active constellation extension followed by a filter prove to be promising towards practical realization of power-efficient transmitters.
\end{abstract}

Keywords: Digital broadcasting, DRM30, OFDM, PAPR reduction, Power amplifier efficiency

\section{Introduction}

The DRM system has been designed particularly to enable a high-quality digital transmission over the current analogue radio broadcast bands using the globally existing frequency and bandwidth plan. DRM can be used for a variety of audio contents with the capacity of integrating text and data. It also offers substantial benefits to information broadcast in case of disasters and emergencies. DRM deploys Coded Orthogonal Frequency Division Multiplexing (COFDM) transmission technology. The DRM system specification such as transmitter and receiver structures, coding rates, and constellations has been licensed and released by the European Telecommunications Standards Institute (ETSI). To support robust

*Correspondence: sanam.moghaddamnia@ikt.uni-hannover.de 'Leibniz Universität Hannover, Institute of Communications Technology, Appelstr. 9A, 30167 Hannover, Germany

Full list of author information is available at the end of the article data transmission at different rates and channel conditions, different operating modes are specified by the DRM standard, which can be divided mainly in DRM30 and $\mathrm{DRM}+[1]$. The latter is designed to operate in VHF bands (30-300 MHz) including the analogue frequency modulation (FM) band, whereas DRM30 modes are developed to work in the bands below $30 \mathrm{MHz}$ like the analogue amplitude modulation (AM). DRM30 has a variety of modes (A, B, C, and D) allowing different spectrum usage settings and is within scope of investigation. Owing to reliable and high data transmission rates OFDM is an attractive communications technology. However, a major problem of OFDM systems is that OFDM signals exhibit high PAPR and thus being subject to nonlinear effects of RF front ends. To achieve maximum power efficiency and coverage in DRM-based systems, a linear high power Amplifier (HPA) is required. However, if the linear range of the HPA is not sufficient or used inefficiently, large 
PAPR leads to high out-of-band emissions and modulation error rate (MER). In other words, unless the HPA is not operated in its linear range with large power backoff, it is impossible to increase the output power without violating the frequency mask and minimum threshold necessary for MER. This results in low power efficiency and expensive transmitters $[1,2]$. This paper is motivated by the need arose to determine efficient PAPR reduction approaches being in compliance with system structure and constraints imposed by the DRM standard. From the practical standpoint, an in-depth investigation on the actual performance of PAPR reduction technologies has not yet been concluded and thus is the subject matter of our research.

In order to improve the power efficiency of OFDM systems, various PAPR reduction techniques have been proposed, which can be categorized into signal scrambling and signal distortion technologies. Signal distortion schemes such as clipping, windowing and peak cancellation reduce peak power at cost of reduced signal quality and usually spectral regrowth. Some signal scrambling schemes such as coding require additional bits to reduce PAPR at the cost of reduced data rate. Another group of signal scrambling approaches including Selective Level Mapping (SLM), Partial Transmit Sequence (PTS), and Hadamard Transformation either requires side information at the receiver to decode the input signal or modification of the receiver structure; thus, both are not standards compliant. Active constellation extension is another signal scrambling scheme, where the outer points of signal constellations are extended adaptively so that the PAPR of an OFDM symbol is minimized [3-5]. This method does not result in throughput and MER loss and thus is of interest for practical use in many OFDM-based systems.

The contributions of this paper are organized as follows; Section 2 gives a brief review of the OFDM signal characteristic and PAPR formulation. Section 3 presents the DRM transmitter structure and specification including criteria used to choose and to implement PAPR reduction schemes. In Section 4, we investigate the feasibility of techniques applied for PAPR reduction. Section 5 provides an efficiency comparison of all respective schemes in terms of PAPR, MER, compliance with frequency mask, and synchronization accuracy. In Section 6, a number of measurements are performed to verify the capability of PAPR reduction scheme based on ACE. Finally, the main issues of this work are concluded in Section 7.

\section{OFDM signal characteristic}

The complex envelope of an OFDM symbol in discretetime domain can be written as

$$
x[n]=\frac{1}{\sqrt{N}} \sum_{k=0}^{N-1} X_{k} e^{\frac{j 2 \pi n k}{N}}, 0 \leq n \leq N-1,
$$

where $N$ is the number of sub-carriers and $X_{k}$ is the data carried by the $k$ th sub-carrier [6]. The PAPR per OFDM symbol can be defined as

$$
\operatorname{PAPR}(x[n])=\frac{\max _{0 \leq n \leq N-1}\left[|x[n]|^{2}\right]}{P_{a v}},
$$

where $P_{a v}=E\left[|x[n]|^{2}\right]$ is the average power of $x[n]$ and $E[\cdot]$ denotes the expectation. Due to the superposition of the numerous independent sub-carriers of random phase and amplitude, OFDM signals usually exhibits high PAPR values. Therefore, the characteristic of the OFDM signal in terms of PAPR distribution should be given in evaluating the performance of any PAPR reduction scheme. The distribution of PAPR bears stochastic characteristics and thus usually is expressed in terms of the Complementary Cumulative Distribution Function (CCDF), indicating the probability that PAPR exceeds a predefined threshold $\gamma$ $(\operatorname{Pr}[\mathrm{PAPR}>\gamma])$. Note that the passband PAPR is about twice of the baseband PAPR considered in our investigation [6].

When measuring the quality of a digital signal, we can not solely rely on the analogue measurement of carrier to noise ratio $(\mathrm{C} / \mathrm{N})$. Although $(\mathrm{C} / \mathrm{N})$ measurements are mathematically coupled to bit-error rate (BER), it cannot provide us with the complete picture. Therefore, the quality of OFDM signals can be directly quantified by measuring the modulation quality via MER. MER is a figure of metric typically defined in the broadcasting industry and determines how much margin the system has before failure. Unlike $\mathrm{C} / \mathrm{N}$ measurements, MER comprises not only noise but also all internal and external interferences and non-linearity impairments introduced to digital signals. MER is specified as a power ratio in $\mathrm{dB}$ by

$$
\operatorname{MER}=10 \log _{10} \frac{\sum_{k=0}^{N-1}\left(I_{k}^{2}+Q_{k}^{2}\right)}{\sum_{k=0}^{N-1}\left(\left(I_{k}-\tilde{I}_{k}\right)^{2}+\left(Q_{k}-\tilde{Q}_{k}\right)^{2}\right)},
$$

where $I_{k}$ and $Q_{k}$ are the ideal I/Q components and $\tilde{I}_{k}$ and $\tilde{Q}_{k}$ are the actual I/Q components of the data symbol of the $k$-th sub-carrier [7].

\section{DRM-specific selection criteria of PAPR algorithms}

Prior to selecting an algorithm for PAPR reduction, it is necessary to set selection criteria relevant to the DRM system as below: 
-It is not allowed to violate the DRM standard such as making modifications to receiver structure, data rate, cell-interleaving and pilot cells.

-The quality of the audio signal has to be maintained. This means that the MER should not fall below a predetermined level [8].

- The frequency spectrum mask is required to conform to the DRM standard. The deterioration of spectral properties should remain within a reasonable limit [9].

- Synchronization performance should not be compromised. In other words, executing the PAPR reduction algorithms should not affect the efficiency of synchronization.

In view of aforementioned criteria, there is a limited number of feasible possibilities to integrate a PAPR reduction module in the DRM transmitter structure [10].

\section{Compatibility of PAPR reduction schemes with DRM system}

A number of different techniques of PAPR reduction has been reported in the literature. A distinction is mainly drawn between signal distortion and signal scrambling techniques. First, we briefly outline the main principle behind these techniques. Subsequently, it is reviewed whether the PAPR reduction approaches are compatible with the DRM system requirements that the standard demands.

\subsection{Signal distortion}

Techniques base on the signal distortion reduce high peaks by non-linearly distorting the OFDM signal. Due to the simple implementation together with the high PAPR gains, an approach based on signal distortion has become a favored PAPR reduction technology. According to literature, the major drawback of these techniques lies in the BER performance degradation [11]. However, it is possible to improve the BER performance via upsampling and conditional filtering as reported in [6]. In the following, we provide a brief overview of PAPR reduction algorithms based on the signal distortion.

\subsubsection{Nonlinear companding transform}

Different companding schemes have been proposed in [11] and [12], each having advantageous in terms of PAPR reduction or spectral properties. Here, the companding noise is regarded as a major problem.

\subsubsection{Peak reduction carrier}

Here, the PAPR reduction is enabled using a portion of subcarriers served as peak reduction carriers, the socalled bearing peak reduction carriers, at the cost of transmission efficiency [13].

\subsubsection{Random phase shuffling}

This approach brings about a PAPR reduction by allocating a random phase to each subcarrier which without corresponding receiver results in a substantial deterioration of BER performance [14].

\subsubsection{Envelop scaling}

This method facilitates the PAPR reduction by adjusting the envelope of a couple of subcarriers. However, this scheme is merely applicable to PSK-modulated signals [11].

\subsubsection{Clipping and filtering}

The simplest approach of PAPR reduction is clipping and filtering, where the signal peak amplitudes exceeding an allowable level are clipped [6]. It should be noted that only the signal amplitude is corrected and the signal phase remains unchanged. However, such a amplitude limiting results in in-band and out-of-band signal distortion and in poor system performance in terms of MER and spectral properties. A trade-off is needed between the PAPR reduction gain and the requirements on MER. According to literature, there are some methods to combat the out-of-band radiation. Applying a bandpass filter after clipping is a simple way to reduce out-of-band distortions, although this leads to peak regrowth and affects the clipping efficiency adversely. To avoid this, one possibility is to use a prefiltering and post-clipping structure. Peak windowing is another improved clipping scheme which uses a multiplicative narrow-band window such as Gaussian to reduce PAPR with minimized out of band radiation as below $[15,16]$ :

$$
x_{c}[n]=x[n]\left(1-\sum_{l} a_{l} w(n-l)\right),
$$

where $x[n]$ and $x_{c}[n]$ are the DRM and the clipped signal, respectively. $w[n]$ is the applied window function and $a_{l}$ the weighting coefficient. The longer the window the more samples are affected, which in turn leads to MER deterioration. Another way to perform clipping with enhanced spectral properties is the peak cancellation scheme [17]. Here, the signal peaks need to be detected first. The next step is to generate pulses which are aligned to each peak. The clipping is performed using a cancellation function which is derived from the sum of the pulses generated beforehand according to

$$
x_{c}[n]=x[n]-\sum_{l} b_{l} w(n-l) .
$$

Here, $b_{l}$ is the peak scaling function and $w(n)$ represents the aligned pulse cancellation function at the position $l$. Ideally, a sinc function can be used as the pulse function. The efficiency of this approach depends on time and frequency characteristics of the pulse function [11]. 
Using peak windowing or cancellation results in less out-band spectral pollution and better PAPR gains without a need for filtering. However, it should be noted that due to the spectral property of DRM signal shown in Fig. 1, it is necessary to use filtering in order to met the DRM-specific frequency mask. Therefore, utilizing peak windowing or cancellation may not be fully fit for purpose.

\subsection{Signal scrambling}

Signal scrambling techniques differ from each other in how the signal scrambling is performed to decrease the PAPR. Reducing PAPR via signal scrambling causes no inband interferences and MER degradation. However, most of these approaches imply the use of a portion of subcarriers/bits or transmission of additional information. Furthermore, to obtain the optimum PAPR reduction gain a search of all possible combinations of the bits is needed which requires a high computational effort [11]. In the following, a brief outline of the signal scrambling schemes is given.

\subsubsection{Hadamard transform}

This approach with a requirement of applying Hadamard and inverse Hadamard Transformation at the transceiver promises a PAPR-reduction of about $2 \mathrm{~dB}$ without affecting the spectral efficiency [18].

\subsubsection{Dummy sequence insertion}

As the name suggest, the PAPR reduction is achieved via a dummy sequence such as complementary and correlation sequences before inverse fast Fourier transformation (IFFT), which should be removed at the receiver side [11].

\subsubsection{SLM and PTS}

Selective Level Mapping (SLM) and Partial Transmit Sequence (PTS) schemes require transmission of additional information as well as the modification of the DRM receiver (De-mapper) [6].

\subsubsection{Interleaving and block coding}

Both schemes are based on similar principles to SLM and PTS and require corresponding de-interleaving and de-coding at the transmitter [11].

\subsubsection{Tone reservation}

Here, unused carriers are deployed to generate a time signal which is added to the original signal. Effective PAPR reduction is provided if sufficient sub-carriers are available [6].

\subsubsection{Tone insertion}

The PAPR reduction is performed here via expanding the constellation points, the so-called polar or rectangular mapping and accordingly the corresponding de-mapping is required at the receiver [6].

\subsubsection{Active constellation extension}

This method provides an attractive solution to combat high PAPR and poor power efficiency. The basic idea is similar to the tone insertion approach, except that the peak reduction is carried out by extending the outer points of signal constellations within given constraints. Therefore, there is no need for de-mapping [5]. This approach is also advantageous in terms of spectral efficiency and applicable to M-QAM/M-PSK modulated

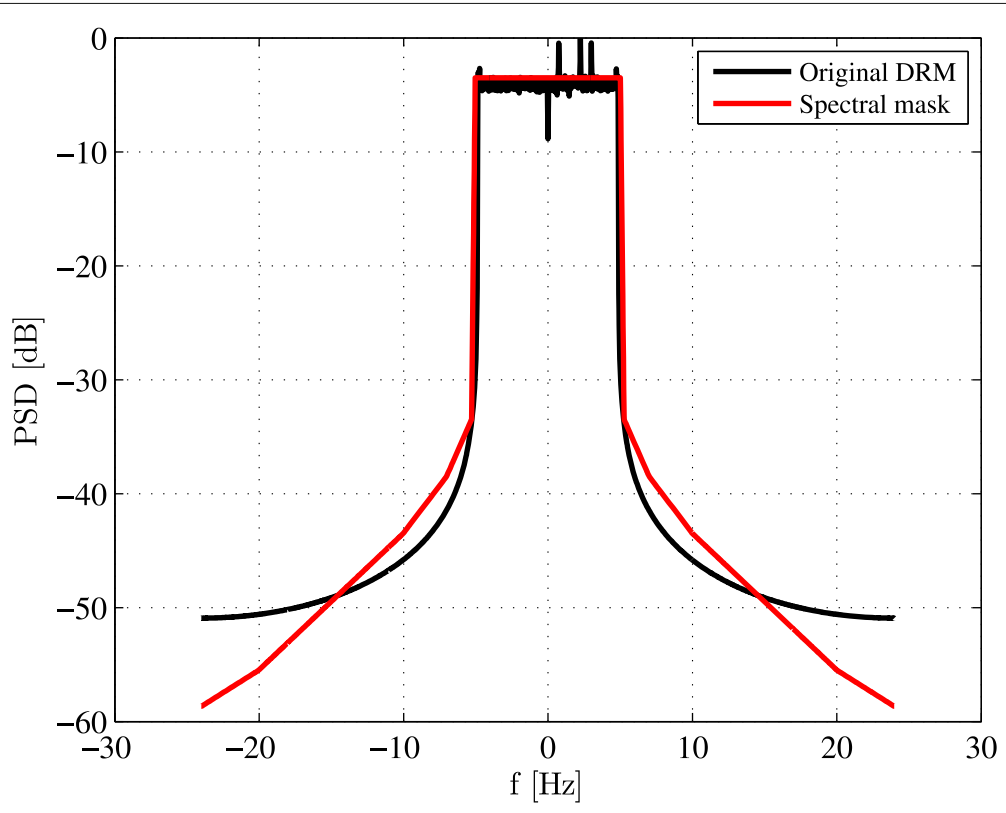

Fig. 1 DRM spectral properties 
signal. As a side effect, an increase in the average transmission power is reported in the literature, which does not matter in practice due to the limitation of signal amplitude and power at the modulator. However, such a limitation results in compression of the constellation points and thus SNR loss at the receiver. Due to the simplicity and efficiency, PAPR reduction using ACE scheme is mainly based on approaches leading to a suboptimal solution. [19] proposes practical methods of projection onto convex sets (POCS) and smart-gradient-project (SGP) for realizing ACE. The first method delivers an optimal solution towards a low PAPR but has a slow convergence of about 7 iterations. The second one provides a fast convergence of approximately three iterations to a minimum PAPR using an adaptive step size. For the DRM system, we consider the POCS leading to the optimal solution. Here, the basic idea is to extend the outer points of signal constellation with a constraint that the minimum Euclidean distance between all points is maintained. Given that the data symbols $X_{k}$ of an assigned constellation, the modified OFDM symbol can be obtained by

$$
\hat{X}_{k}=X_{k}+C_{k} \text {, }
$$

where $C_{k}$ is the extension vector for the given sub-carrier constellation and subject to the above mentioned constraint. In other words, only the components of $C_{k}$ within an allowable extension area are considered. This is carried out iteratively until PAPR is reasonably minimized. In summary, the algorithm works as follows. Data symbols of a given sub-carrier $X_{k}$ are transformed into a time-domain signal $x$ using IFFT. Any $|x[n]|>C L$ is clipped in magnitude and the clipped portion can be obtained by $c[n]=$ $(C L-|x[n]|) e^{j \varphi[n]}$. Using FFT $c[n]$ is transformed into the extension vector $C_{k}$. The algorithm is iterated until PAPR is essentially decreased or a given iteration time is reached. Another issue concerns the clipping level choice being of particular importance to achieve the best PAPR reduction. Specifying the ideal clipping level is a difficult task, because this depends on various factors as reported in [19].

All methods of PAPR reduction described above are not applicable to DRM systems. The reason for this is that many of these schemes are tied to special requirements which do not comply with the DRM standard and system architecture. The compatibility check in Table 1 shows that approaches based on clipping and filtering or ACE and filtering do not violate the DRM-specific selection criteria and can be deployed for DRM systems.

\section{Simulation results}

In order to determine the most efficient PAPR reduction approach for the DRM, we tested potential schemes with real I/Q signals generated by a DRM modulator at the sampling frequency of $f_{s}=48 \mathrm{kHz}$. We evaluated the often-used DRM30 in robustness mode B with $10-\mathrm{kHz}$ bandwidth and $N=206$ subcarriers, where 64-QAM and 4-QAM mapping are used for the MSC and the FAC/SDC, respectively. Both realization and evaluation of all signal processing methods were carried out in MATLAB. The performance evaluation includes PAPR, distance to frequency mask and synchronization accuracy analysis. The CCDF curves are based on $10^{5}$ random baseband OFDM symbols. Notice that at least an average MER of about $35 \mathrm{~dB}$ at the transmitter side is tolerable for high quality data transmission. Therefore, the PAPR reduction is subject to the condition that the minimum MER and DRM-specific frequency mask are met. Finally, the influence of the utilized schemes on the synchronization is investigated.

\subsection{PAPR analysis}

For an accurate PAPR estimation of continuous-time OFDM signals, usually the signal should be oversampled at least by a factor of 4 [6]. The applied sampling frequency of DRM modulator amounts to $f_{s}=48 \mathrm{kHz}$ (approx. 5 times oversampled) and thus is sufficient enough to evaluate the efficiency of PAPR reduction techniques for DRM signals. We investigate the efficiency of all PAPR reduction schemes applicable to DRM at a CCDF of $10^{-4}$. It is noticeable that all parameters of the PAPR reduction schemes are adjusted specifically for DRM30 mode B. The clipping level is adjusted so that an average MER-value of about $35 \mathrm{~dB}$ is granted for each scheme, whereas the spectral properties of signal are maintained. As the efficiency of the ACE approach greatly depends on the used clipping level, first, the PAPR reduction performance and the induced SNR loss for different clipping levels are investigated. The evaluation results in Fig. 2 show that the best trade-off between the SNR loss and the PAPR reduction performance can be achieved at a clipping level $C L=$ $7.8 \mathrm{~dB}$ above the average power, which results in a slight increase in average SNR loss of about $0.28 \mathrm{~dB}$. A further point is that the efficiency of the considered methods depends on the filter design. Due to the baseband system evaluation, a real and linear phase low-pass equiripple FIR-filter with a DRM-adapted magnitude response is utilized for both post-filtering and pre-filtering approaches (see Table 2).

The results in Fig. 3 show that both clipping and windowing in combination with postfiltering provide low PAPR reduction gains of $14-9.5=4.5 \mathrm{~dB}$ and $14-$ $8.2=5.8 \mathrm{~dB}$, respectively. As supposed, the windowing scheme promises only a moderate PAPR improvement of $5.8-4.5=1.3 \mathrm{~dB}$ in comparison to the clipping approach. The pre-filtering and post-clipping issues deliver the best PAPR reduction gain of $14-7=7 \mathrm{~dB}$, whereas the difference between the achievable average PAPR of $6.35 \mathrm{~dB}$ and maximum PAPR recorded amounts to $7-6.35=0.65 \mathrm{~dB}$. 
Table 1 DRM-compatible PAPR reduction schemes

\begin{tabular}{llll}
\hline Technique & Method & $\begin{array}{l}\text { Standard } \\
\text { conformity }\end{array}$ & Explanation \\
\hline Signal distortion & Nonlinear companding transform & No & Need for modification of the receiver \\
& Peak reduction carrier & No & Need for modification of the transceiver \\
& Random phase shuffling & No & Need for modification of the receiver \\
& Envelop scaling & No & Applicable only to PSK-modulated signals \\
& Clipping and filtering & Yes & No need for modifications \\
Signal scrambling & Hadamard transform & No & Need for modification of the receiver \\
& Dummy sequence insertion & No & Need for modification of the receiver \\
& SLM and PTS & No & Need for modification of the receiver \\
& Interleaving and block coding & No & Need for modification of the transceiver \\
& Tone reservation & No & Need of Additional subcarriers \\
& Tone insertion & No & Need for modification of the receiver \\
& ACE & Yes & No need for modifications
\end{tabular}

The ACE postfiltering scheme provides slightly less PAPR reduction gain of $14-7.7=6.3 \mathrm{~dB}$, but it offers an improved average PAPR value of about $5.6 \mathrm{~dB}$ regardless of the filter parameter settings, which concurrently involves robustness against spectral pollutions. Therefore, $\mathrm{ACE}$ is seen to have a great potential for reducing PAPR based on DRM. [20] shows that by combining ACE and clipping approaches slightly better performance in terms of quality of service or equivalent noise degradation can be achieved in comparison with the sole use of ACE, albeit at the cost of slight deterioration in the PAPR gain.

\subsection{Spectral property}

Another issue needing consideration is to verify whether the processed signal is within the limits of the DRM spectral mask. In other words, the amount of out-ofband distortion induced by applying PAPR reduction technologies has to be kept within certain limits to prevent DRM-specific frequency mask violations. Figure 4 illustrates the average power spectral density (PSD) of the original DRM and processed signals with regards to the default spectral mask. It should be noticed that the postfiltering has no adverse effect on the achievable PAPR by ACE and thus there is no need to exclude constellations of outer carriers to remove the out-band distortions, which in turn reduces degree of freedom for the extension and PAPR gain. According to the results the out-of-band distortions can be removed by means of an appropriate adjustment of the applied filter parameter. It is known that the amount of nonlinear distortion depends on the
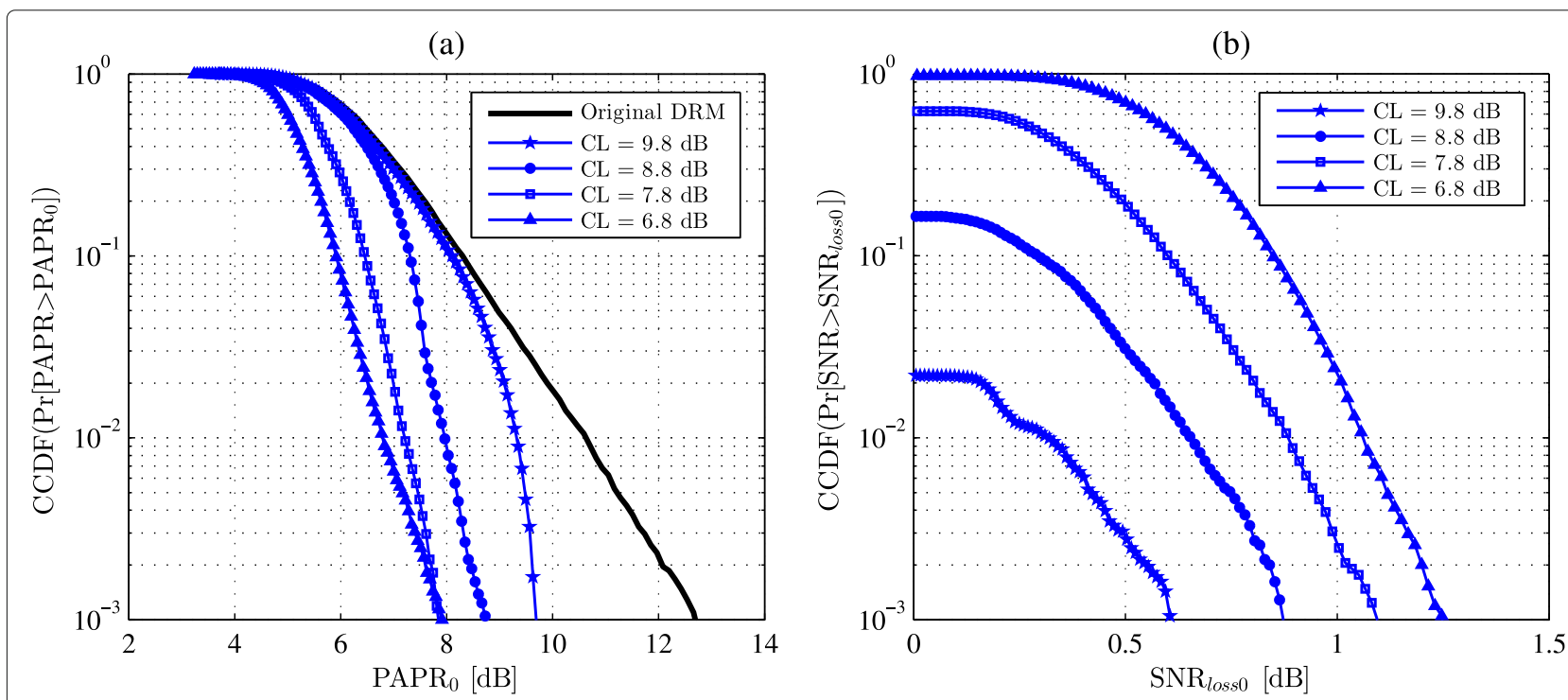

Fig. 2 ACE performance in terms of distribution of PAPR (a) and SNR loss (b) for different clipping levels 
Table 2 Specification of the low-pass equiripple FIR-filter

\begin{tabular}{lll}
\hline Parameter & Prefilter & Postfilter \\
\hline Length [sample] & 0.1 & 0.2 \\
Passband edge $[\mathrm{kHz}]$ & 8.3 & 4.7 \\
Stopband edge $[\mathrm{kHz}]$ & 8.7 & 5.2 \\
Passband ripple $[\mathrm{dB}]$ & 0.001 & 0.001 \\
Stopband atten. $[\mathrm{dB}]$ & 60 & 15
\end{tabular}

input back-off (IBO) given also by PAPR. It should be noticed that the PAPR values are not directly related to the so-called output back-off, which is required for a practical power amplifier to sustain an allowable level of out-of-band radiation (OBO). In fact, the amount of tolerable spectral pollution is higher for a lower PAPR gains achieved by PAPR reduction technologies as shown in [21] and this is a further reason to decide for the ACE approach.

\subsection{Synchronization efficiency}

How the PAPR reduction algorithms affect the efficiency of synchronization is a further important question so far left untreated within PAPR reduction studies. In this work, we mainly focus on the synchronization schemes proposed for DRM, where the receiver has the full knowledge of the pilot cell position and phase. During the acquisition phase the receiver performs a search for a known pilot pattern of each OFDM symbol by calculating the correlation between the known pilot pattern $P$ and the received OFDM Symbols $R$.
To obtain a robust frame synchronization the search procedure for pilot positions can be carried out over multiple OFDM symbols $N_{\text {sync }}$ as below:

$M(n, c)=\frac{1}{N_{\text {sync }}} \sum_{l=0}^{N_{\text {sync }}-1}$

$$
\left|\sum_{m \in \Gamma[(n-l) \bmod N]} P_{(n-l) \bmod N}^{*}(m) R_{i-l}(m+i)\right|,
$$

where $0 \leq n \leq N_{s}$ and $-F_{c} \leq c \leq F_{c}$ are time and frequency sample indexes used to identify the symbol position in OFDM transmission frame. Here, $F_{c}$ is the maximum frequency offset to be expected and $N_{s}$ presents the number of OFDM symbols per a frame. $m$ is an auxiliary variable representing the discrete frequency index from the pilot index range $\Gamma$ available for the OFDM symbol in question and specified for each DRM Mode distinctly. $i$ is the time index of OFDM symbols, of which the position is to be determined. It should be noted that $M(n, c)$ can achieve the peak value at $n$ and $c$ for which the received sequence contains the pilot sequence. In order to detect the begin of the OFDM frame the merit factor (MF) is introduced as follows [22, 23]:

$$
\mathrm{MF}=\frac{M^{2}(n, c)}{\sum_{c=-F_{c}}^{F_{c}} M^{2}(n, c)} .
$$

The merit factor gives the ratio of the main lobe to the side lobes energy of the correlation and serves as a

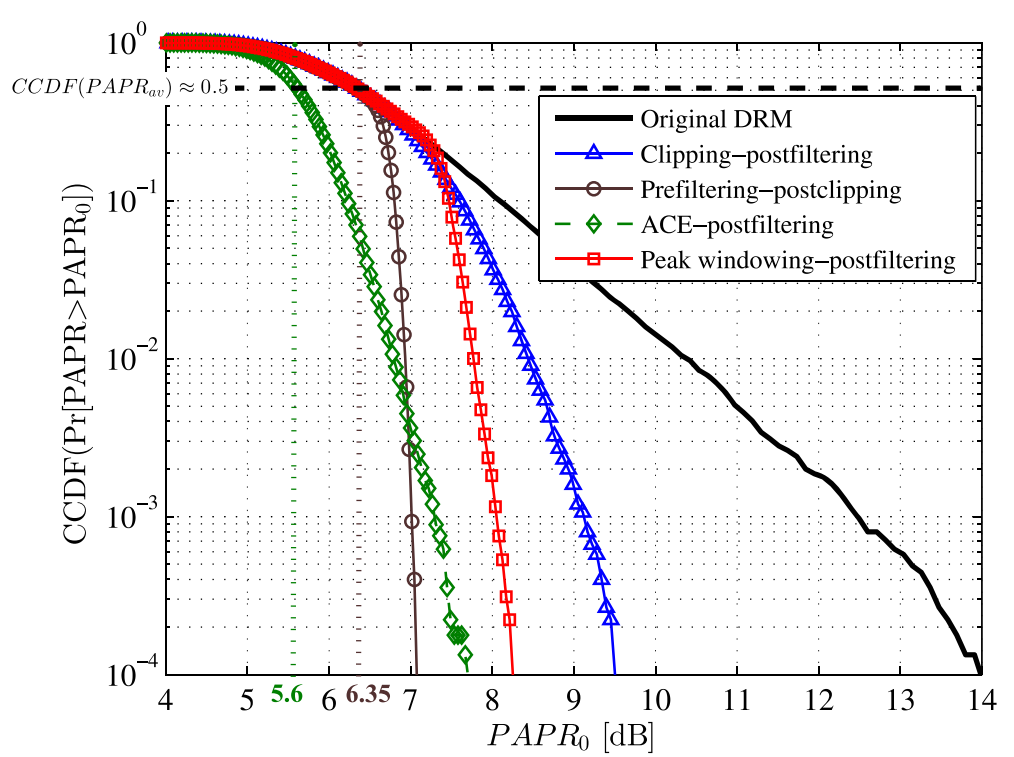

Fig. 3 Comparison of PAPR CCDF for different PAPR reduction schemes. The best performance in terms of PAPR reduction is achieved by the prefiltering-postclipping and ACE postfiltering 


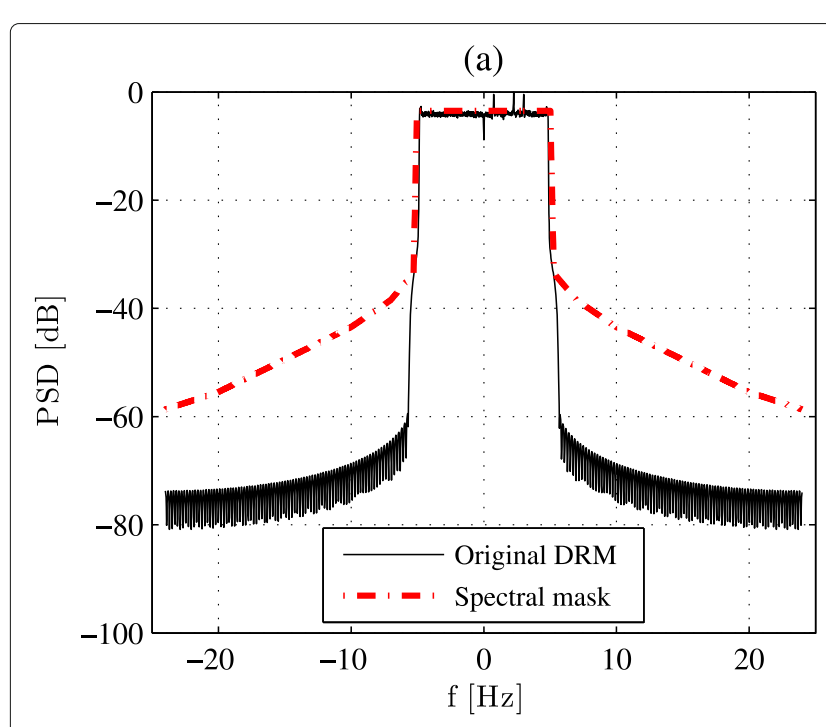

(c)

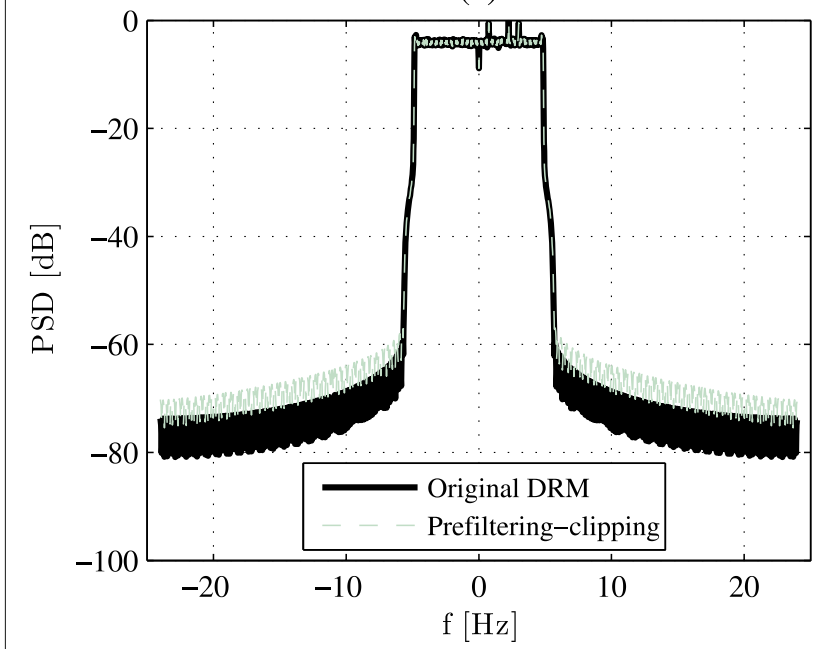

(b)

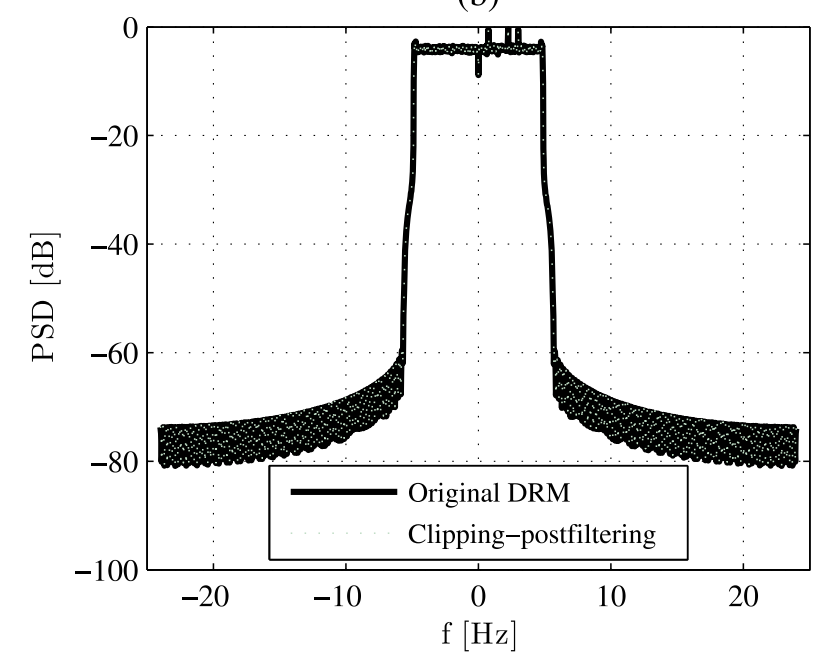

(d)

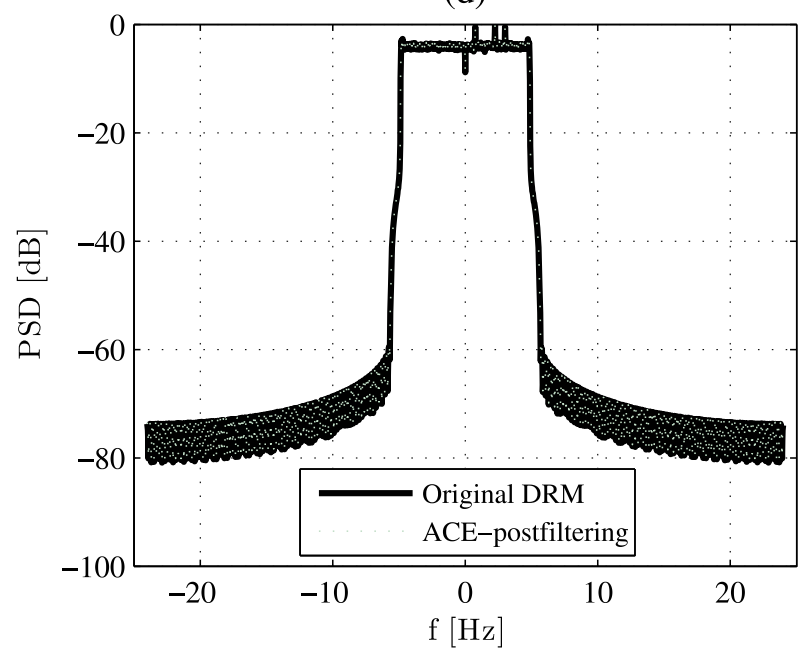

Fig. 4 The effect of PAPR reduction on the the spectral properties of the DRM signal. a shows a comparison between the postfiltered original DRM signal and the spectral mask, $\mathbf{b}, \mathbf{c}$, and $\mathbf{d}$ compare the postfiltered original DRM signal with processed signals via PAPR reduction schemes

measure of the correlation quality. Hence, By detecting the maximum merit factor $\mathrm{MF}_{\max }$ over all OFDM symbols the start of the transmission frame can be found. An efficient synchronization is feasible, if the merit factor reaches the maximum value at the beginning of the OFDM frame and for all other positions has a small value. In order to determine the effect of PAPR reduction algorithms on the synchronization performance, we calculate the mean squared error (MSE) between the merit factor of original and PAPR reduced DRM signals as

$$
\mathrm{MSE}=E\left[\left(\mathrm{MF}_{\mathrm{org}}-\mathrm{MF}_{\mathrm{PAPR}}\right)^{2}\right],
$$

where $\mathrm{MF}_{\text {org }}$ and $M F_{\mathrm{PAPR}}$ are the MF of the original and PAPR reduced DRM signal, respectively.

Table 3 compares the effect of proposed PAPR reduction methods on MF via the resulted MSE. As seen, there is no significant deterioration of MF due to the PAPR reduction. However, the correlation quality for the ACE processed signal is slightly reduced as a result of extending outer constellation points to comparable values of pilot cell magnitudes. In summary, the synchronization performance is not unaffected by the processing done for the PAPR reduction.

Table 3 The results of MSE calculated for the MF of original and PAPR reduced signals

\begin{tabular}{ll}
\hline Method & MSE $[\mathrm{dB}]$ \\
\hline Clipping-postfiltering & -83 \\
Prefiltering-postclipping & -88 \\
ACE postfiltering & -59
\end{tabular}




\subsection{Power efficiency}

Depending on the coverage area, the DRM transmit power can amount to several hundred kilowatt. As reported in [24], the efficiency of HPA can be given by the portion of supply power $P_{\mathrm{DC}}$ that is delivered to the load as $\eta=\frac{P_{\text {out }}}{P_{\mathrm{DC}}}$ where $P_{\text {out }}$ is the average output power. Assuming an ideal linear model for HPA, we obtain $\eta=\frac{1}{2 \mathrm{PAPR}}$. The impact of PAPR reduction on the power can be specified as average power trade-offs by

$$
E\left[P_{\text {out }}\right]=\frac{P_{\mathrm{DC}}}{2 E[\mathrm{PAPR}]},
$$

or

$$
E\left[P_{\mathrm{DC}}\right]=2 P_{\text {out }} E[\mathrm{PAPR}] .
$$

Accordingly, the PAPR reduction results in either power savings with a fixed $P_{\text {out }}$ or increased transmit output power having a fixed $P_{\mathrm{DC}}$. It is often desired to improve the coverage and thus $P_{\text {out }}$ having a fixed $P_{\mathrm{DC}}$. Based on the evaluation results of PAPR at a CCDF of $10^{-4}$, the HPA efficiency of the original DRM signal is about $2 \%$, whereas the one achieved by the most promising schemes, prefiltering-postclipping and ACE postfiltering, is about 10 and $8.5 \%$, respectively.

\section{Measurements}

To evaluate the transmit power gain of the ACE and postfiltering, which is determined as the most promising PAPR reduction approach, a number of measurements were performed on a DRM test platform provided by RFmondial. Measurement results in Fig. 5 illustrate the filtered original DRM signal without being transmitted over the antenna (without HPA). The result window in the upper left side of Fig. 5 demonstrates the full compliance of DRM signal with the specific requirement on the frequency mask. The signal power and distance to frequency mask are about -4 and $-11.2 \mathrm{~dB}$, respectively. The constellation diagram at the upper right side shows the high quality of demodulated data, which is also reflected in the measurement of SNR as a function of sub-carrier. It should be noticed that the SNR value seen at the lower left corner of the figure is measured in time domain, whereas the graphics window of SNR is the result of frequency domain measurements over sub-carriers. For channels only subject to additive white Gaussian noise (AWGN), SNR corresponds to the value of MER (see Fig. 5).

Measurements in Figs. 6 and 7 were carried out to assess the power efficiency in terms of increased transmit output power. For this purpose, the filtered original DRM signal and the PAPR reduced signal, processed by the ACE and postfiltering algorithm are fed over the Ampegon 1 kW DRM SW transmitter amplifier to the transmitting antenna and then at the receiver end, are demodulated by the DRM demodulator for thorough performance monitoring. As seen in the spectrum windows, both original and PAPR reduced signals comply with frequency mask regulations. However, we can see that the output power achieved by PAPR reduced signal is approximately $10 \mathrm{~dB}$ higher than that provided by the original signal and without having nonlinear distortions in the amplifier. It should be emphasized that the MER in Fig. 7 does not reflect the actual value, since the symbols modified by ACE are also considered for the calculation of MER and lead to faulty values. This is clearly to be recognized from the modulation characteristics of demodulated data in the associated constellation diagram.

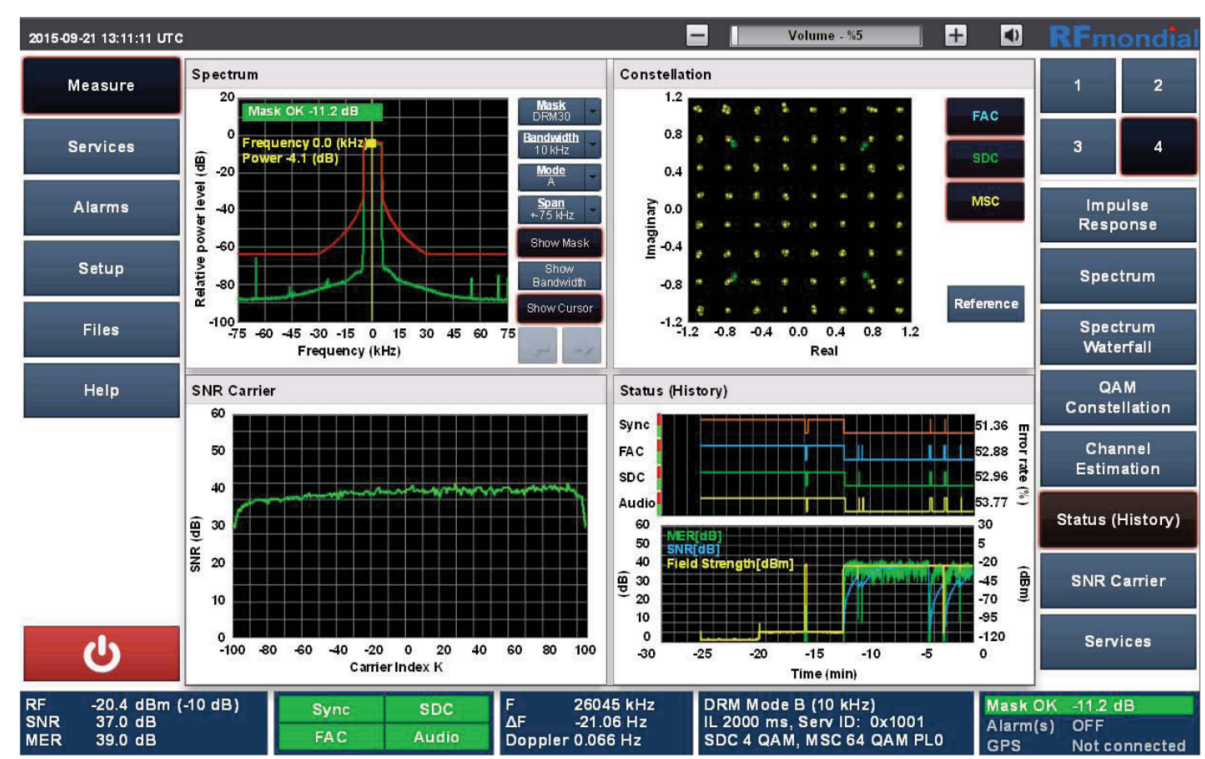

Fig. 5 Evaluation based on DRM test setup for the original DRM signal after filtering and without HPA 


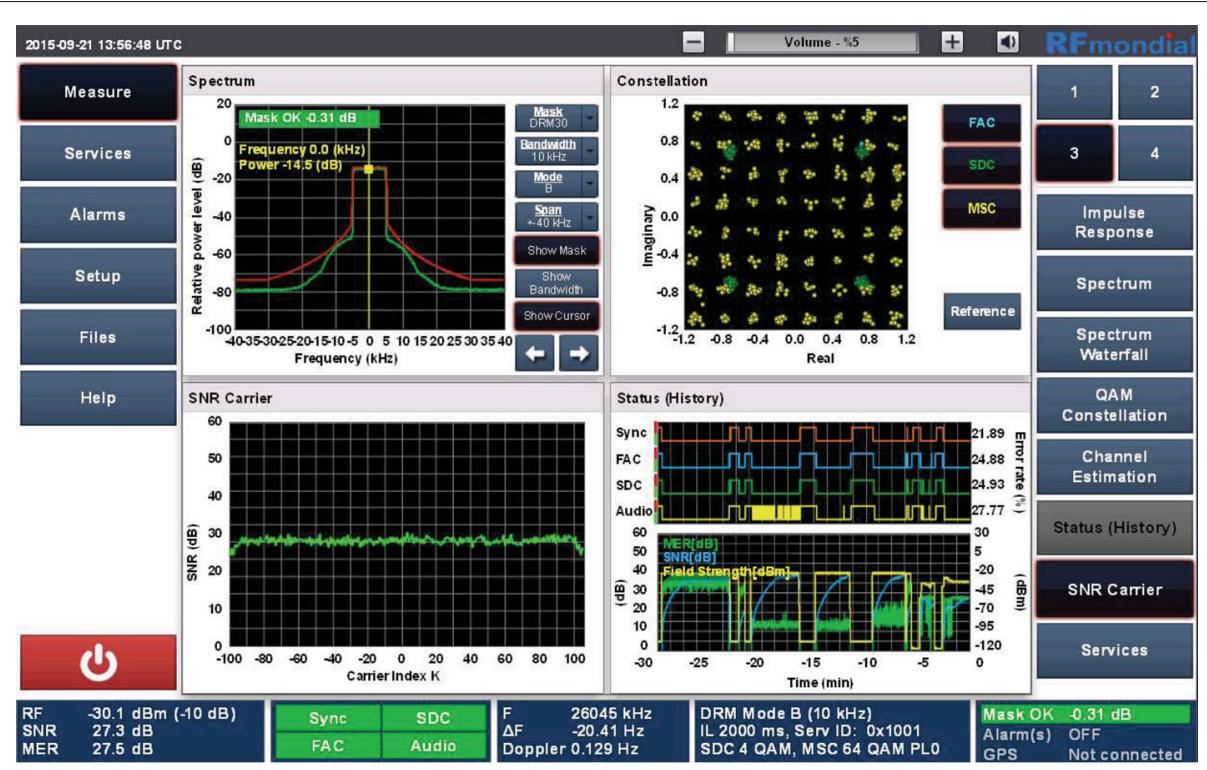

Fig. 6 Evaluation based on DRM test setup for the original DRM signal after filtering and with HPA

\section{Conclusions}

The main focus of this work is on identifying DRMcompatible PAPR reduction schemes and their actual efficiency in real operation. On the basis of criteria prescribed by the DRM standard, all PAPR reduction schemes applicable to DRM are determined and the underlying parameters are adjusted individually to achieve the best trade-off between the PAPR gain, MER loss, spectral pollution, and synchronization accuracy. According to our investigation, the ACE postfiltering approach provides the superior performance in terms of average PAPR value, robustness to spectral pollution, and modulation quality. The simple procedure of prefiltering and postclipping promises a superior PAPR gain in comparison to schemes based on the postfiltering structure and thus serves as another alternative to reduce PAPR. Moreover, it is possible to achieve almost equal amount of maximum and average PAPRs via this approach, which implies

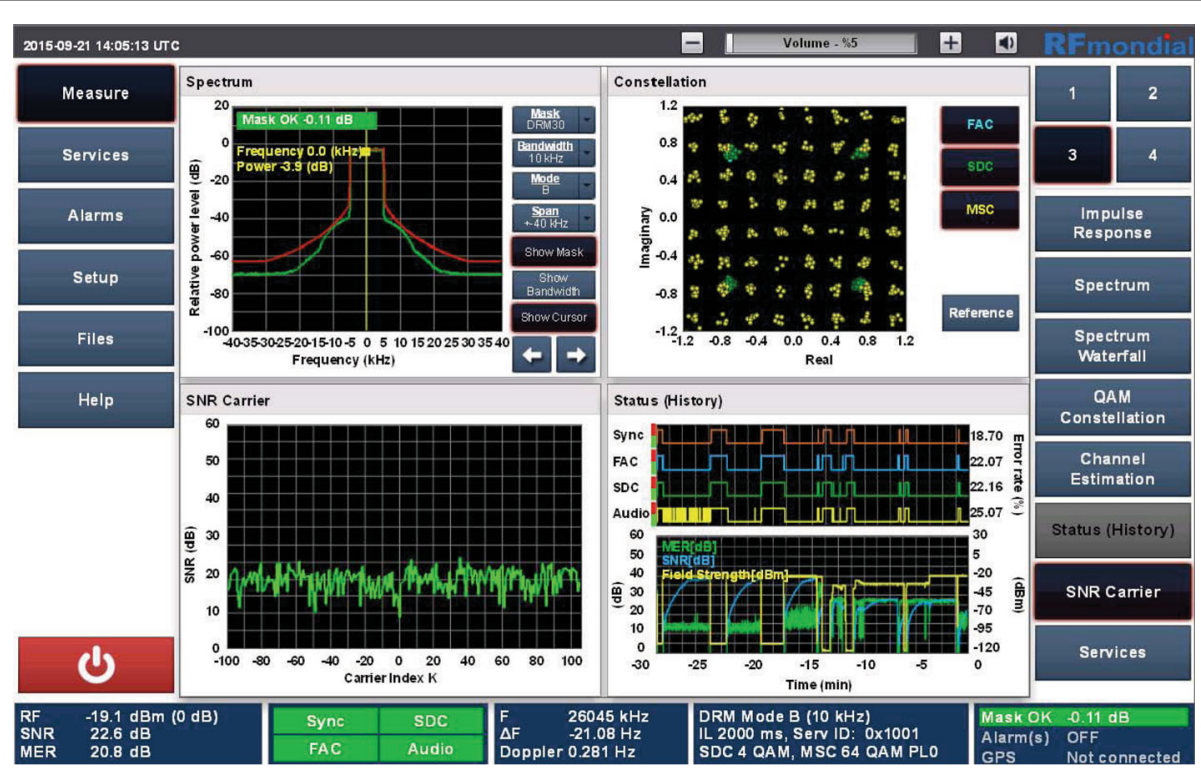

Fig. 7 Evaluation based on DRM test setup for the DRM signal processed by ACE after filtering and with HPA 
that the occurrence of non-linearities can be entirely prevented by the proper adjustment of amplifier operation range. However, the PAPR gain achieved by the prefiltering and post-clipping other than the ACE postfiltering strongly depends on the applied filter properties. In other words, adjusting the filter parameters to achieve compliance with the frequency mask results in PAPR deterioration. Furthermore, none of these approaches have any impact on the synchronization efficiency. All this taken together, both approaches are worth considering from a practical point of view for PAPR reduction in DRM systems. According to our investigations, the ACE postfiltering is seen as the most promising PAPR reduction approach applicable to DRM, which has been verified by measurements. In particular, combining the ACE postfiltering scheme and the issues of adaptive equalization of amplifier nonlinearities bears a great potential for green broadcasting and is subject of our future work.

\section{Competing interests}

The authors declare that they have no competing interests.

\section{Author details \\ ${ }^{1}$ Leibniz Universität Hannover, Institute of Communications Technology, Appelstr. 9A, 30167 Hannover, Germany. ${ }^{2}$ RFmondial GmbH, Appelstr. 9A, 30167 Hannover, Germany.}

Received: 22 April 2016 Accepted: 5 October 2016

Published online: 26 October 2016

\section{References}

1. DRM Consortium, The DRM Digital Broadcasting System Introduction and Implementation Guide Grand-Saconnex, Revision 2, 2013, (Geneva, 2013). http://www.drm.org/wp-content/uploads/2013/09/DRM-guide-artwork9-2013-1.pdf

2. B Jaksić, M Petrović, P Spalević, R Ivković, S Minić, Digitalization of radio through DRM standard on mediumwave and shortwave. Int. J. Eng. Innovative Technol. (IJEIT). 3(9), 72-78 (2014)

3. T-D Chiueh, P-Y Tsai, OFDM Baseband Receiver Design for Wireless Communications. (John Wiley \& Sons (Asia) Pte Ltd, 2007). https://books. google.de/books?id=vhKkLimLrQoC

4. R Prasad, OFDM for Wireless Communications Systems. (Artech House, 2004). https://books.google.de/books?id=gVE9vkreKWMC

5. SH Han, JH Lee, An overview of peak-to-average power ratio reduction techniques for multicarrier transmission. IEEE Wireless Commun. 12(2), 56-65 (2005)

6. T Jiang, Y Wu, An overview: peak-to-average power ratio reduction techniques for OFDM signals. IEEE Trans. Broadcasting. 54(2), 257-268 (2008)

7. JTJ Penttinen, The LTE-Advanced Deployment Handbook: The Planning Guidelines for the Fourth Generation Networks. (John Wiley \& Sons, 2016). https://books.google.de/books?id=H67QCgAAQBAJ

8. ETSI, EN 302 245-1 V1.1.1 (2005 01) Electromagnetic compatibility and Radio spectrum Matters (ERM). Transmitting equipment for the Digital Radio Mondiale (DRM) broadcasting service. Part 1: Technical characteristics and test methods

9. ETSI, EN 302 245-2 V1.1.1 (2005 01) Electromagnetic compatibility and Radio spectrum Matters (ERM). Transmitting equipment for the Digital Radio Mondiale (DRM) broadcasting service. Part 2: Harmonized EN under article 3.2 of the R\&TE Directive

10. ETSI, ES 201980 V3. 1.1 Digital Radio Mondiale (DRM): System Specification (2009)

11. V Vijayarangan, R Sukanesh, An overview of techniques for reducing peak to average power ratio and its selection criteria for orthogonal frequency division multiplexing radio systems. J. Theor. Appl. Inform. Technol. $\mathbf{5}(1), 25$ (2009)

12. X Huang, JH Lu, JL Zheng, KB Letaief, J Gu, Companding transform for reduction in peak-to-average power ratio of OFDM signals. IEEE Trans. Wireless Commun. 3(6), 2030-2039 (2004)

13. CE Tan, IJ Wassell, Data bearing peak reduction carriers for OFDM systems, Proceedings of the 2003 Joint Conference of the Fourth International Conference on Information, Communications and Signal Processing, 2003 and the Fourth Pacific Rim Conference on Multimedia Sinsapore, vol. 2, (2003), pp. 854-858

14. H Nikookar, KS Lidsheim, Random phase updating algorithm for OFDM transmission with low PAPR. IEEE Trans. Broadcasting. 48(2), 123-128 (2002)

15. R Van Nee, A Wild, Reducing the peak-to-average power ratio of OFDM, Vehicular technology conference, 1998, VTC 98, 48th IEEE, Vol. 3. (IEEE, 1998), pp. 2072-2076

16. M Pauli, H-P Kuchenbecker, Minimization of the intermodulation distortion of a nonlinearly amplified OFDM signal. Wireless Pers. Commun. 4(1), 93-101 (1997)

17. TMay, H Rohling, Reducing the peak-to-average power ratio in OFDM radio transmission systems, 48th IEEE Vehicular Technology Conference (VTC 98), Ottawa, Ont., vol. 3, (1998), pp. 2474-2478

18. M Park, J Heeyong, N Cho, D Hong, C Kang, PAPR reduction in OFDM transmission using Hadamard transform. IEEE Int. Conf. Commun. 1, 430-433 (2000)

19. BS Krongold, DL Jones, PAR reduction in OFDM via active constellation extension. IEEE Int. Conf. Acoustics, Speech Signal Process. 4, 525-528 (2003)

20. M Lanoiselee, $\mathrm{H}$ Lin, A powerful and real time PAPR reduction process for energy efficiency improvement in OFDM communication systems, (11th International Symposium on Wireless Communications Systems (ISWCS), Barcelona, 2014), pp. 159-164

21. S Singh, MS Kumar, HS Mruthyunjaya, Effect of peak-to-average power ratio reduction on the multicarrier communication system performance parameters. Int. J. Electrical Comput. Eng. 3(4), 779-786 (2009)

22. H-P Kuchenbecker, W Schäfer, Frame and frequency synchronisation for coherent OFDM systems based on pilots with pseudorandom phase, (6th International OFDM-Workshop (InOWo), Hamburg, 2001), pp. 171-175

23. A Waal, Konzeption und Realisierung eines digitalen Hörfunksystems mit Mehrwertdiensten zur lokalen Versorgung. (Dissertation, Leibniz Universität Hannover, 2010)

24. AK Gurung, FS Al-Qahtani, AZ Sadik, ZM Hussain, Power savings analysis of clipping and filtering method in OFDM systems, (Australasian Telecommunication, Networks and Applications Conference (ATNAC). Adelaide, 2008), pp. 204-208

\section{Submit your manuscript to a SpringerOpen ${ }^{\circ}$ journal and benefit from:}

- Convenient online submission

- Rigorous peer review

- Immediate publication on acceptance

- Open access: articles freely available online

- High visibility within the field

- Retaining the copyright to your article

Submit your next manuscript at springeropen.com 